This item was submitted to Loughborough's Institutional Repository (https://dspace.lboro.ac.uk/) by the author and is made available under the following Creative Commons Licence conditions.

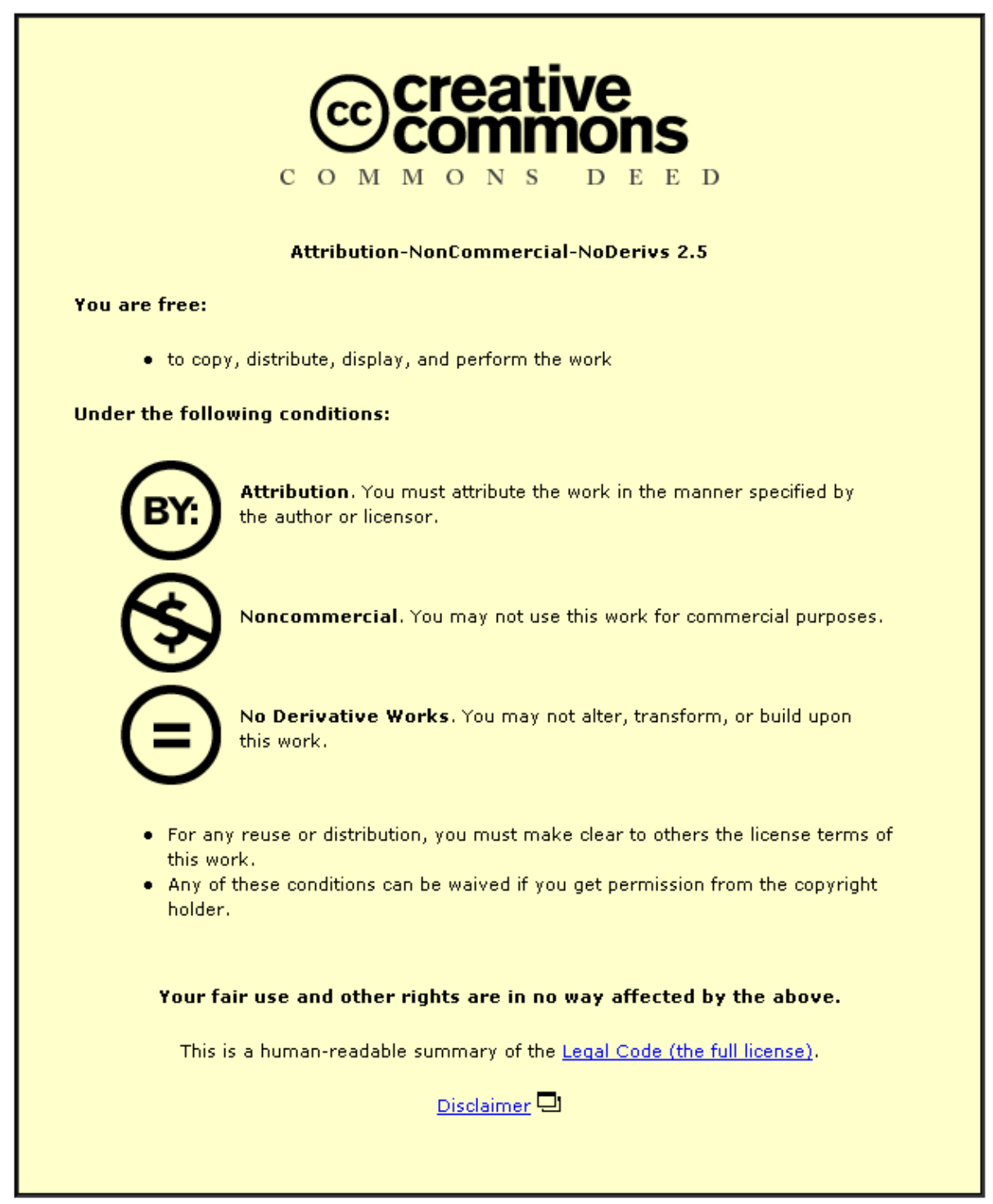

For the full text of this licence, please go to: http://creativecommons.org/licenses/by-nc-nd/2.5/ 


\title{
ON THE EFFICIENT ESTIMATION OF BLOOD VELOCITIES
}

\author{
Zhuo Zhang ${ }^{\dagger}$, Andreas Jakobsson ${ }^{\ddagger}$, Malcolm D. Macleod*, Jonathon A. Chambers ${ }^{\dagger}$ \\ $\dagger$ The Centre of Digital Signal Processing, Cardiff School of Engineering \\ Cardiff University, Cardiff CF24 0YF, UK. \\ $\ddagger$ Department of Electrical Engineering, Karlstad University, Sweden \\ * QinetiQ Ltd, St. Andrew's Road, Malvern, WR14 3PL, UK.
}

\begin{abstract}
Pulsed wave (PW) Doppler ultrasound systems are commonly used to examine blood flow dynamics and the technique plays a very important role in numerous diagnostic applications. Commonly, narrow-band PW systems estimate the blood velocity using an autocorrelation-based estimator. Herein, we examine a recently proposed hybrid frequency estimator, and via extensive numerical simulations using simulated blood scatterers show the achievable performance gain of this method as compared to the traditional approach.
\end{abstract}

\section{INTRODUCTION}

Ultrasound systems for the estimation of blood velocities have found numerous diagnostic applications and are currently widely used by medical doctors [1]. Today, more than one fourth of all medical imaging studies are performed using ultrasound, and the proportion is ever increasing [2]. The great majority of commercially available PW system use a statistically inefficient narrow-band autocorrelationbased technique developed by Kasai et al [3] (see also [4]). It is both numerically robust and computationally simple $[5,6]$, but suffers from a limited estimation accuracy, especially for lower signal to noise ratios (SNR). In this paper, we examine the possible performance gain achievable by instead using the recently proposed computationally and statistically efficient hybrid implementation [7]. As shown in [7], the hybrid estimator, which requires only $\mathcal{O}(N)$ operations, outperforms other recently proposed statistically efficient frequency estimation approaches, lowering the SNR at which the Cramér-Rao lower bound (CRLB) is reached. Furthermore, it was shown that the performance of the hybrid estimator is essentially unaffected by the underlying frequency. Herein, we briefly summarize the hybrid estimator and via extensive numerical simulations examine its performance as compared to the traditional autocorrelationbased estimator for both synthetic sinusoidal data as well as simulated radio frequency $(\mathrm{RF})$ data from simulated blood scatterers obtained using the Field II program [8].

\section{THE HYBRID ESTIMATOR}

As is well-known, the backscattered signal resulting from a blood flow along the transmission direction (normally called the axial flow) can be expressed as [9]

$$
y(t)=\beta e^{i(\omega t+\theta)}+n(t),
$$

for $t=0, \ldots, N-1$, where $\beta \in \mathbb{R}, \omega$ and $\theta \in[0,2 \pi)$ denote the deterministic but unknown amplitude, frequency, and initial phase, respectively, of a complex sinusoid. Here,

$$
\omega=2 \pi \frac{2 v_{z} f_{c}}{c}
$$

where $v_{z}$ denotes the velocity component of the backscattering flow, $f_{c}$ the carrier frequency in emission, and $c$ the speed of propagation in the tissue. Further, $n(t)$ is an additive noise which, for simplicity, is often modeled as a circular zero mean complex white Gaussian noise. As suggested in [10], the data model in (1) can be written as

$$
y(t)=[1+v(t)] \beta e^{i(\omega t+\theta)},
$$

where $v(t)=\beta^{-1} n(t) e^{-i(\omega t+\theta)}$ is a complex white sequence. Let $v_{r}(t)$ and $v_{i}(t)$ denote the real and the imaginary parts of $v(t)$, respectively. For high SNR,

$$
1+v(t) \approx e^{i \arctan v_{i}(t)} \approx e^{i v_{i}(t)},
$$

allowing the approximation $y(t) \approx \beta e^{i \phi(t)}$, where $\phi(t)=$ $\omega t+\theta+v_{i}(t)$. Thus, it is possible to estimate the phase as

$$
\begin{aligned}
\Delta \phi(t) & \triangleq \arg \left[y^{*}(t) y(t+1)\right] \\
& \approx \omega+v_{i}(t+1)-v_{i}(t)
\end{aligned}
$$

where $(\cdot)^{*}$ denotes the complex conjugate, suggesting the autocorrelation estimator $[3,4]$

$$
\hat{\omega}_{c}=\arg \left[\frac{1}{N-1} \sum_{t=0}^{N-2} y^{*}(t) y(t+1)\right] \text {. }
$$


To remove the typical frequency dependence of the SNR threshold (which is caused by the wraparound of angle measurements at $\pm \pi$ ), we form a downshifted signal using $\hat{\omega}_{c}$, i.e.,

$$
y_{d}(t)=y(t) e^{-i \hat{\omega}_{c} t} .
$$

Further, to reduce the SNR threshold, we apply a $K$-tap averaging filter, forming

$$
y_{f}(t)=\frac{1}{K} \sum_{k=0}^{K-1} y_{d}(t+k) .
$$

Similar to (5), the adjacent phase difference of (8) can be formed as

$$
\Delta \phi_{f}(t)=\arg \left[y_{f}^{*}(t) y_{f}(t+1)\right]=\omega_{f}+u_{c}(t),
$$

where, for $K=2$, (see [7] for a general $K$ )

$$
u_{c}(t)=\frac{v_{i}(t+2)-v_{i}(t)}{2 \cos (\omega / 2)} .
$$

It is worth noting that the noise process $u_{c}(t)$ will now be coloured due to the average filtering and the correlation of input noise components between $\Delta \phi_{f}(t)$ and $\Delta \phi_{f}(t+1)$. To further reduce the SNR threshold, we apply a recent outlier detection scheme proposed in [11]. Here, $\pm 2 \pi$ outliers are detected if $\left|\beta_{t}\right|>\left|\beta_{t-1}\right|,\left|\beta_{t}\right|>\left|\beta_{t+1}\right|$ and $\left|\beta_{t}\right|>\lambda$, with

$$
\beta_{t}=\Delta \phi_{f}(t-1)+\Delta \phi_{f}(t)+\Delta \phi_{f}(t+1),
$$

where $\beta_{-1}=\beta_{N-K}=0$ and $\Delta \phi_{f}(-1)=\Delta \phi_{f}(N-K)=$ 0 . Thus, the outliers can be removed as follows

$$
\Delta \tilde{\phi}_{f}(t)= \begin{cases}\Delta \phi_{f}(t)-\operatorname{sign}\left(\beta_{t}\right) 2 \pi & \text { if outlier detected } \\ \Delta \phi_{f}(t) & \text { otherwise }\end{cases}
$$

for $t=0, \ldots, N-K-1$. Here, both $K$ and $\lambda$ are user parameters; in the simulations below, we use $K=6$ and $\lambda=4$ (see [12] for a further study on the influence of both $K$ and $\lambda$ ). After the SNR threshold reduction using (9) and (12), further improvement can be achieved by taking into account the colouration of the noise term in (9). It can be shown that a frequency correction term, $\hat{\omega}_{f}$, can be found as [13]

$$
\hat{\omega}_{f}=\sum_{t=1}^{(N-K) / K} q(t) \sum_{m=1}^{K-1} \Delta \tilde{\phi}_{f}(t K-m),
$$

where

$$
q(t)=\frac{6 t K(N-t K)}{N^{3}-N K^{2}},
$$

with $t=1,2, \ldots,(N-K) / K$. Combined with the coarse estimate, the hybrid frequency estimate is found as

$$
\hat{\omega}_{h}=\hat{\omega}_{c}+\hat{\omega}_{f}
$$

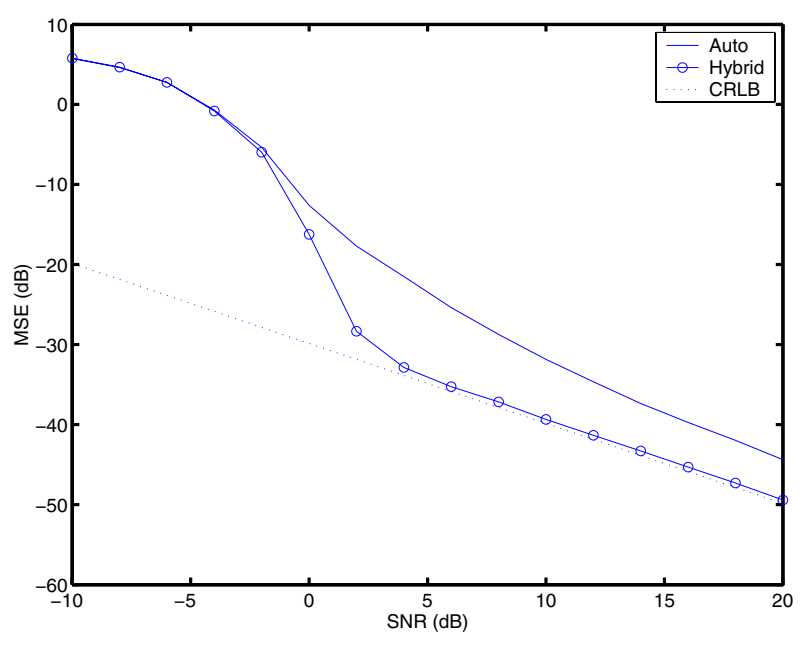

Figure 1: The MSE of the examined estimators as a function of the SNR, for $v_{z}=0.5 \times v_{N y q}$, with synthetic data.

In summary, the proposed hybrid estimator is found by first forming the downshifted signal in (7) using the estimate in (6). Then, the phase difference of the filtered signal is formed using (9), followed by the outlier removal scheme in (12). Finally, the refined frequency estimate is formed as (15), using (13).

\section{SIMULATIONS - SYNTHETIC DATA}

In this section, we will briefly examine the performance of the proposed estimator. Initially, we consider $N=18$ data samples containing a single complex sinusoid with frequency $\omega=0.5 \pi$, corresponding to half of the maximum detectable axial velocity, the so-called Nyquist velocity, here denoted $v_{N y q}$, and examine the estimated mean square error (MSE) as a function of the SNR. Here, the simulation results have been obtained using $10^{4}$ Monte-Carlo simulations. Figure 1 illustrates the MSE for the hybrid estimator, as compared to the standard autocorrelation estimator (termed Auto in the Figures) and the corresponding CRLB as given in [14]. The figure clearly shows that the autocorrelation estimator is not statistically efficient for any examined SNR. Further, it can be seen that the hybrid estimator is statistically efficient, reaching the CRLB (which indicates a theoretical lower bound on the achievable variance of any unbiased estimator) at a low SNR threshold.

\section{SIMULATIONS - RF DATA}

In this section, we examine the performance of the hybrid estimator for simulated blood scatterers using the Field II program [8]. Initially, we simulate a single fibre-like flow 


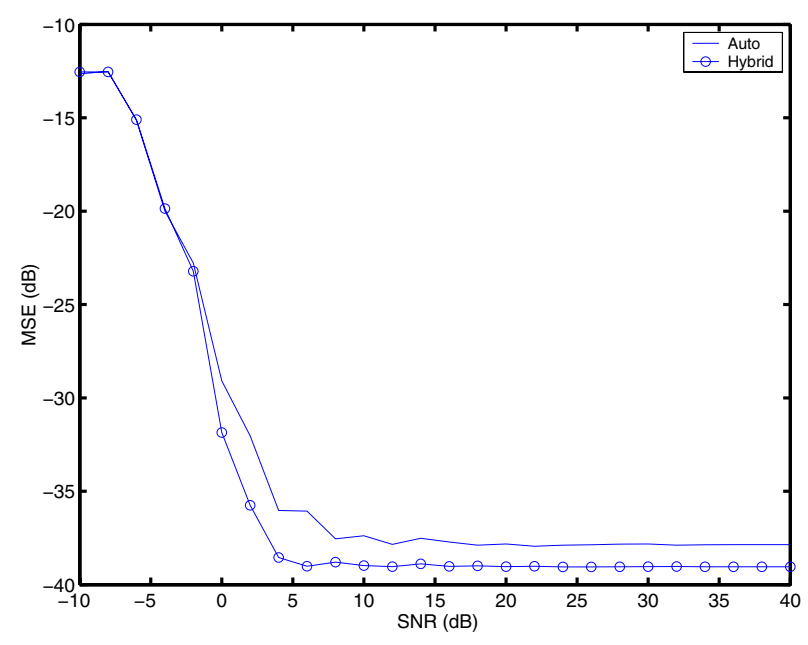

Figure 2: The MSE of the examined estimators as a function of the SNR, for $v_{z}=0.5 \times v_{N y q}$, with fibre RF data.

which comprises 1000 blood particles lying along the vessel center and moving towards the transducer along the beam direction (pure axial velocity) with constant velocity $v_{z}=$ $0.5 \times v_{N y q}$. The details of the parameters for simulating the RF data are listed in Table 1. Figure 2 shows the MSE of the examined estimators as a function of the SNR at the transmission focus which is $35 \mathrm{~mm}$ away from the transducer surface. As is seen from the figure, the hybrid estimator uniformly exhibits better performance than the autocorrelation estimator over the whole examined SNRs. Further, we consider a laminar blood flow (consisting of $3 \times 10^{5}$ scatters) with parabolic profile, i.e.,

$$
v(r)=\left(1-r^{2}\right) v_{0},
$$

where $r \in[0,1]$ is the relative radius, and $v_{0}$ the velocity at the vessel center. Here, $v_{0}=0.8 \times v_{N y q}$, again using the parameters given in Table 1. As seen in Figure 3, the hybrid estimator shows preferable performance also for this case. As the data generation is somewhat time-consuming, we have limited the simulations for both the fibre and parabolic flow to only 100 Monte-Carlo simulations. Finally, to verify the performance of the hybrid estimator in a somewhat more realistic situation, the RF data simulating the flow in the carotid artery are examined, taking into account the tissue motion due to the breathing and pulsation ${ }^{1}$. The upper plot in figure 4 shows one of the RF lines. The dominant peaks on both sides of the plot depict the strong clutter effects due to the existence of vessel walls (represented by the dashed vertical lines) and the tissue motion. To enable high quality blood velocity estimation, efficient clutter filter must be applied prior to the velocity estimation. In this

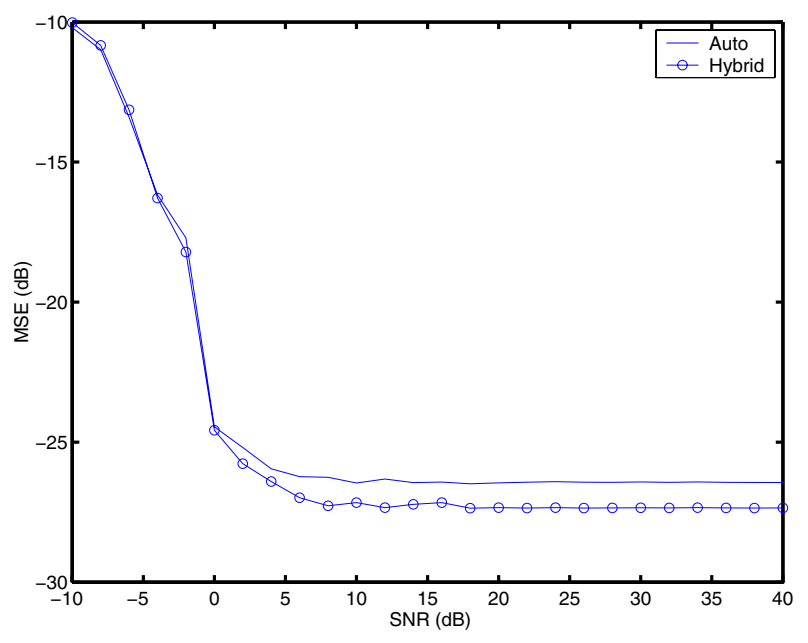

Figure 3: The MSE of the examined estimators as a function of the SNR, for $v_{z}=0.5 \times v_{N y q}$, with parabolic flow RF data.

Table 1: Field II parameters

\begin{tabular}{|l|l|}
\hline Carrier frequency, $f_{c}$ & $10 \mathrm{MHz}$ \\
\hline Sampling frequency, $f_{s}$ & $100 \mathrm{MHz}$ \\
\hline Pulse repetition frequency, $f_{p r f}$ & $10^{4} \mathrm{~Hz}$ \\
\hline Velocity in fibre, $v$ & $0.5 \times v_{N y q}$ \\
\hline Peak velocity in parabolic flow, $v_{0}$ & $0.8 \times v_{N y q}$ \\
\hline Sound speed, $c$ & $1540 \mathrm{~m} / \mathrm{s}$ \\
\hline RF lines per estimate, $N$ & 18 \\
\hline Pulse oscillation (M) & 10 \\
\hline Doppler angle in fibre & $0^{\circ}$ \\
\hline Doppler angle in parabolic flow case & $45^{\circ}$ \\
\hline Bandwidth & 0.6 \\
\hline Center of vessel & $35 \mathrm{~mm}$ \\
\hline Vessel radius & $5 \mathrm{~mm}$ \\
\hline Transducer type & Phased array \\
\hline Transducer elements & 64 \\
\hline Transducer element pitch & $0.077 \mathrm{~mm}$ \\
\hline Transducer element kerf & $0.00385 \mathrm{~mm}$ \\
\hline Transducer element width & $0.732 \mathrm{~mm}$ \\
\hline Transducer element height & $5 \mathrm{~mm}$ \\
\hline Focus in transmission & $35 \mathrm{~mm}$ \\
\hline Focus in reception & $35 \mathrm{~mm}$ \\
\hline Elevation focus & $25 \mathrm{~mm}$ \\
\hline Apodization in transmission & Hanning \\
\hline Apodization in reception & Hanning \\
\hline
\end{tabular}

\footnotetext{
${ }^{1}$ Data available at: http: / /www. oersted.dtu.dk/31655/ ?ultrasound_data/sim_car_wall.html
} 
work, a second order eigenfilter is used (see [15] for further details). The lower plot in the figure illustrates the estimated velocity profile crossing the whole vessel using the autocorrelation method and the proposed hybrid estimator, respectively. As seen from the figure, both the autocorrelation method and the hybrid estimator accurately estimate the velocities close to the vessel walls. However, close to the vessel center, the hybrid estimator clearly outperforms the autocorrelation method. It is worth noting that the center of the profile is not accurately estimated. This is likely an effect of the fixed order clutter filter; in the center of the vessel, the filter order should typically be lower than at the vessel walls as discussed in [15].
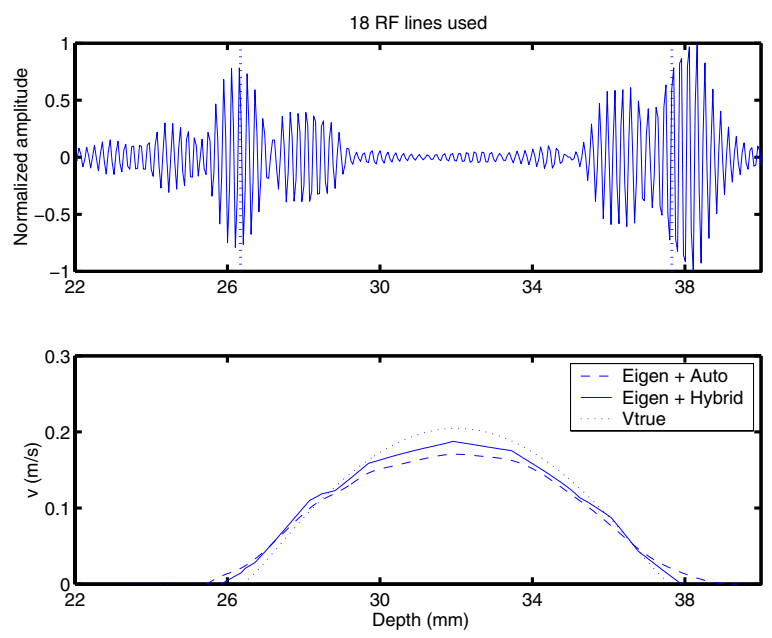

Figure 4: The estimated velocity profile using RF data from simulated carotid artery.

\section{ACKNOWLEDGEMENT}

The first author is grateful to Prof. Peter Wells at Cardiff University, U.K., for interesting and stimulating discussions.

\section{REFERENCES}

[1] J. A. Jensen, "Range/Velocity Limitations for Timedomain Blood Velocity Estimation," Ultrasound in Med. and Biol., vol. 19, pp. 741-749, 1993.

[2] WFUMB, "World Federation for Ultrasound in Medicine and Biology News, vol.4, no.2," Ultrasound in Med. and Biol., vol. 23, pp. 974, 1997.

[3] C. Kasai, K. Namekawa, A. Koyano, and R. Omoto, "Real-time Two-dimensional Blood Flow Imaging Using an Autocorrelation Technique," IEEE Trans. Sonic Ultrason, vol. 32, pp. 458-464, 1985.
[4] G. W. Lank, I. S. Reed, and G. E. Pollon, "A Semicoherent Detection and Doppler Estimation Statistic," IEEE Trans. Aerospace and Electronic Systems, vol. 9, no. 2, pp. 151-165, March 1973.

[5] P. N. T. Wells, "Ultrasonic Colour Flow Imaging," Phys. Med. Biol., vol. 38, no. 39, pp. 2113-2145, Dec. 1994.

[6] S. K. Alam and K. J. Parker, "Implementation Issues in Ultrasonic Flow Imaging," Ultrasound in Med. and Biol., vol. 29, no. 4, pp. 517-528, 2003.

[7] Z. Zhang, A. Jakobsson, M. D. Macleod, and J. A. Chambers, "A Hybrid Phase-based Single Frequency Estimator," IEEE Signal Processing Letters, to appear.

[8] J. A. Jensen, "Field: A Program for Simulating Ultrasound Systems," in Medical and Biological Engineering and Computing, 1996, vol. 34, pp. 351-353.

[9] J. A. Jensen, Estimation of Blood Velocities Using Ultrasound, Cambridge University Press, New York, 1996.

[10] S. A. Tretter, "Estimating the Frequency of a Noisy Sinusoid by Linear Regression," IEEE Trans. Information Theory, vol. 31, no. 6, pp. 832-835, Nov. 1985.

[11] M. D. Macleod, “An Improved Fast Estimator of the Frequency of a Single Complex Tone," in Sixth IMA International Conference on Mathematics in Signal Processing, Cirencester, UK, 2004, IMA, pp. 155158.

[12] Z. Zhang, A. Jakobsson, M. D. Macleod, and J. A. Chambers, "Statistically and Computationally Efficient Frequency Estimation of a Single Tone," Tech. Rep. EE-2005-02, Dept. of Electrical Engineering, Karlstad Univ., Karlstad, Sweden, February 2005.

[13] M. L. Fowler, "Phase-Based Frequency Estimation: A Review," Digital Signal Processing, vol. 12, no. 2, pp. 590-615, Oct. 2002.

[14] D. C. Rife and R. R. Boorstyn, "Single-Tone Parameter Estimation from Discrete-Time Observations," IEEE Trans. Information Theory, vol. 20, no. 5, pp. 591-598, Sept. 1974.

[15] Z. Zhang, A. Jakobsson, J. A. Jensen, and J. A. Chambers, "On the Efficient Implementation of Adaptive Clutter Filters for Ultrasound Color Flow Imaging," in Sixth IMA International Conference on Mathematics in Signal Processing, Cirencester, UK, 2004, IMA. 